\title{
A EDUCAÇÃO A DISTÂNCIA COMO ALTERNATIVA PARA O ENSINO DA FERRAMENTA COMPUTACIONAL AUTOCAD 2D
}

\author{
PORTO ALEGRE/RS MAIO/2018 \\ $\begin{array}{cl}\text { Paulete Fridman Schwetz } & \text { - UFRGS - pauletefs@gmail.com } \\ \text { Luciana Neves Nunes } & \text { - UFRGS - lunenu@gmail.com } \\ \text { Gabriel Bertaluci da Costa } & \text { - UFRGS - gbcosta96@gmail.com }\end{array}$
}

Tipo: Investigação Científica (IC)

Natureza: Descrição de Projeto em Andamento

Categoria: Métodos e Tecnologias

Setor Educacional: EDUCAÇÃO SUPERIOR

\begin{abstract}
RESUMO
O presente trabalho busca avaliar, a partir de uma análise estatística, os resultados da implementação de um curso à distância para o ensino do programa computacional AutoCAD 2D, com modelo pedagógico desenvolvido especialmente para esse fim. Essa ferramenta é utilizada pela disciplina de Desenho Técnico Instrumentado, oferecida pela Universidade Federal do Rio Grande do Sul. A partir da criação do modelo pedagógico, foi desenvolvido um curso no ambiente virtual de aprendizagem (AVA) MOODLE UFRGS. Finalizado a criação curso, foi iniciado o processo de implementação para sua validação. Para tanto, foram formados três grupos de alunos de graduação de diferentes cursos de Engenharia e Design, com variados perfis e sem conhecimento prévio de AutoCAD. Os resultados obtidos foram analisados, estatisticamente, através da ferramenta computacional SPSS Statistics. A cada implementação, foram realizadas alterações no curso para sua melhoria. As análises estatísticas demonstraram que o desempenho dos alunos que realizaram a última versão do curso à distância foi bastante satisfatório, sendo equiparada ao desempenho dos estudantes que aprenderam a ferramenta de forma presencial. Esses resultados indicam a adequação do curso para o ensino do programa computacional AutoCAD 2D à distância.
\end{abstract}

Palavras-chave: Ensino a distância; Modelo pedagógico; AutoCAD

\section{AGRADECIMENTOS}

AGRADECEMOS À SECRETARIA DE EDUCAÇÃO À DISTÂNCIA (SEAD/UFRGS), AO PROGRAMA DE APOIO À GRADUAÇÃO (PAG/UFRGS) E À UNIVERSIDADE FEDERAL DO RIO GRANDE DO SUL PELO APOIO NO DESENVOLVIMENTO DESTA PESQUISA. 


\section{INTRODUÇÃO}

Nas últimas décadas, as universidades brasileiras estão passando por um processo de mudança significativo em relação aos processos educacionais. Pode-se dizer que há uma crise paradigmática na educação, que exige mudanças nas práticas educativas e, consequentemente, nos modelos pedagógicos. Dentro deste panorama, a Educação a Distância $(\mathrm{EaD})$ surge como uma alternativa bastante atraente. Através desta prática, nasce um novo espaço pedagógico, cujas características são: o desenvolvimento de competências e habilidades, respeito ao ritmo individual, formação de comunidades de aprendizagem, redes de convivência. Assim, conceitos como construção do conhecimento, autonomia, autoria, interação, construção de um espaço de cooperação, respeito mútuo, solidariedade, centrado na atividade do aprendiz, identificação e solução de problemas passam a ser os alicerces deste novo modelo que está emergindo (BEHAR, 2009).

Esta modalidade traz consigo novas possibilidades em termos de qualidade de aprendizado, flexibilidade de tempo para os estudantes, facilidade de acesso de qualquer local com acesso a internet e disponibilidade de educação para locais onde antes não era possível um ensino muito específico e de qualidade (BERNARDI, 2011). Porém, esta nova proposta educacional, diferente da educação presencial, que se caracteriza por uma interação professor-alunos com espaço-tempo definidos, onde predomina a comunicação oral, passa a utilizar o conceito de comunicação multimedial, não exigindo a copresença espacial e temporal. Por isso, surge a necessidade do desenvolvimento de modelos pedagógicos próprios para a $\mathrm{EaD}$, onde não cabe apenas a adaptação de modelos derivados do ensino presencial, mas a construção de práticas que sustentem a gestão da distância pedagógica (BEHAR et al., 2009). Neste contexto geral, pesquisas acerca deste modelo educacional se fazem constantemente necessárias, tanto para a academia quanto para as organizações, a fim de que, conhecendo melhor a EaD, suas potencialidades e riscos, as instituições de ensino, em especial as de ensino superior, possam implementá-la de forma qualificada, proporcionando aprendizado por meio de um ensino de qualidade, que possibilite a preparação profissional tão requerida pelo mercado de trabalho, nos mais diferentes segmentos.

O presente trabalho busca propor um curso à distância para o ensino do programa computacional AutoCAD 2D, a partir da definição de um modelo pedagógico especialmente concebido para este fim. Esta ferramenta, desenvolvida pela Autodesk Inc., é utilizada pela disciplina de Desenho Técnico Instrumentado, oferecida pela Universidade Federal do Rio Grande do Sul aos alunos dos cursos de Engenharia e 
Design.

\section{REFERENCIAL TEÓRICO}

A Educação à Distância caracteriza-se por proporcionar a possibilidade de comunicação multidirecional entre os sujeitos, mesmo quando estes se encontrem distantes espacialmente, devido ao suporte dos recursos tecnológicos utilizados. A interação entre as partes pode ocorrer em tempo real, ou seja, de forma síncrona. Ferramentas síncronas são as que necessitam da coincidência da presença temporal do educador e do educando. Independentemente da localização geográfica dos participantes do curso, existe a obrigatoriedade de que todos estejam conectados, ao mesmo tempo, a um programa que irá gerenciar o contato do grupo. Uma outra forma de comunicação é a interação realizada de ferramentas assíncronas. Essa categoria não exige a presença do educador e do educando simultaneamente. Os acessos poderão ser feitos nos horários disponíveis de cada um dos participantes (MOORE, 2007).

A introdução do Ensino a Distância em disciplinas do ensino superior pressupõe uma nova arquitetura pedagógica constituída por quatro elementos principais: fundamentação do planejamento/proposta pedagógica, preparação do conteúdo, proposição de atividades, interações e procedimentos de avaliação, além da definição de uma plataforma de EaD (BEHAR, 2009).

A fundamentação da proposta pedagógica deve contemplar os propósitos da aprendizagem, a organização do tempo e do espaço, as expectativas na relação de atuação dos participantes, bem como a definição dos direitos e deveres de cada agente (professor ou aluno). Cabe salientar que a adoção desta proposta pressupõe a modificação das competências dos agentes envolvidos em relação ao ensino presencial. Com relação ao papel do professor, o mesmo precisará acompanhar os alunos individualmente, pelas suas trajetórias pedagógicas, pela sua participação em eventos síncronos e assíncronos e pela sua produção. Com tal monitoramento individual, é possível trabalhar com as diferenças, valorizando a riqueza de um grupo heterogêneo de aprendizagem colaborativa. Segundo Moran (2014), ocorre uma mudança na relação de espaço, tempo e comunicação com os alunos. O espaço de trocas se estende da sala de aula para o virtual. O autor enfatiza que o papel do professor passa a ser o de gerente de pesquisa, de estimulador de busca, de coordenador dos resultados. Com relação ao discente, a principal modificação em sua competência é a necessidade de que ele precisa assumir o papel de sujeito do processo de ensino e aprendizagem. Segundo Lapa (2008), para que isso seja possível, o estudante necessita conhecer as novas ferramentas de tecnologia, informação e comunicação (tic's), buscando uma 
apropriação crítica dessas ferramentas, passando a praticar uma aprendizagem ativa. Outra questão importante diz respeito à organização e planejamento do estudo, de modo a preparar as condições necessárias para a aprendizagem e, também, a tirar o máximo de proveito das oportunidades disponibilizadas pela nova proposta de ensino. $A$ autora enfatiza, por outro lado, a importância da disposição do estudante a aprender como aprender à distância.

Em relação ao conteúdo, os materiais instrucionais devem ser cuidadosamente planejados para que a partir deles seja possível construir conhecimento, desenvolver capacidades, competências e habilidades. Os aspectos metodológicos e tecnológicos tratam não somente da seleção das técnicas, procedimentos e dos recursos informáticos a serem utilizados, mas também da relação, articulação e estruturação que a combinação destes elementos terá. Dentre os aspectos tecnológicos, tem-se a Plataforma ou Ambiente Virtual de Aprendizagem (AVA). O AVA é um espaço na Internet formado pelos sujeitos e suas interações e formas de comunicação que se estabelecem através de uma plataforma, cuja estrutura tecnológica é composta por funcionalidades e por uma interface gráfica.

O advento da Educação a Distância traz consigo novas possibilidades em termos de qualidade de aprendizado, flexibilidade de tempo para os estudantes, facilidade de acesso de qualquer local com acesso a internet e disponibilidade de educação para locais onde antes não era possível um ensino muito específico de qualidade. No entanto, a qualidade dos cursos à distância ainda é uma indagação que permanece e, a partir deste trabalho, procurar-se-á definir diretrizes para a implantação da EaD no ensino da ferramenta computacional AutoCAD 2D, para que a mesma apresente um padrão de qualidade equiparável ou superior ao de cursos presenciais.

\section{MÉTODO}

Com base em ampla revisão bibliográfica, foi desenvolvido o modelo pedagógico, cuja estrutura é apresentada na Figura 1.

Figura 1 - Modelo Pedagógico
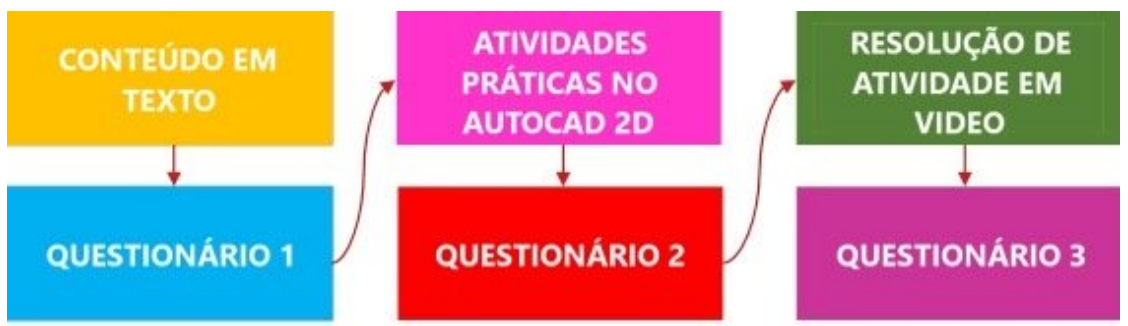
Analisando a Figura 1, observa-se que o aluno encontra três propostas distintas de aprendizado: (i) textos explicativos com ilustrações, através de arquivos gerados em formato pdf, com o intuito de facilitar seu acesso; (ii) atividades práticas a serem desenvolvidas, utilizando a ferramenta computacional AutoCAD (disponível gratuitamente em versão estudantil). Em todas as atividades é proposto um exercício diferenciado, com o intuito de desafiar o usuário a experimentar novas situações; (iii) soluções destas atividades, apresentadas em formato de vídeo, gerado a partir de capturas de tela do AutoCAD. A Figura 1 evidencia que, após o acesso a cada proposta de aprendizagem, o usuário deve responder um questionário, visando verificar a construção de seu conhecimento. Cada questionário libera o avanço no curso, através de um recurso de restrição.

A partir da definição do modelo pedagógico, foi desenvolvido o material instrucional, bem como a criação de atividades, interações e procedimentos de avaliação. A seguir, foi definido o Ambiente Virtual de Aprendizagem. O AVA MOODLE UFRGS foi escolhido para o desenvolvimento do curso. Após concluir o desenvolvimento do curso no AVA, foi iniciado o processo de validação do modelo pedagógico para verificar sua adequação. Três grupos de alunos de graduação de diferentes cursos de Engenharia, com variados perfis e sem conhecimento prévio de AutoCAD, foram formados:

- Grupo 1: Estudantes voluntários, cursando o primeiro semestre do curso de Engenharia;

- Grupo 2: Estudantes do curso de Agronomia, cursando o primeiro semestre. Ficou estabelecido que seu desempenho seria considerado como $10 \%$ da nota final da disciplina;

- Grupo 3: Estudantes matriculados na disciplina de Desenho Técnico II que cursavam Engenharia Mecânica ou Engenharia de Produção. Foi definido que o desempenho dos alunos no trabalho avaliativo, ao final do curso, representaria $15 \%$ da nota final da disciplina.

Durante a realização de cada curso, os estudantes foram monitorados pela equipe formada pelo professor e tutores para solução de problemas, dúvidas e para o controle do avanço dos alunos no curso de forma individual. Após o encerramento do curso, os alunos realizaram um trabalho avaliativo presencial para a verificação da construção de seu conhecimento. Este trabalho avaliativo era composto por duas partes: um questionário de múltipla escolha e um exercício prático utilizando o programa computacional. O exercício prático foi proposto, principalmente, com o intuito de verificar o aprendizado da impressão do desenho no papel. Além disso, os alunos responderam um questionário qualitativo anônimo, cujo objetivo era a realização de uma 
autoavaliação. O mesmo trabalho avaliativo foi realizado com alunos voluntários que aprenderam a ferramenta computacional de forma presencial na disciplina de Desenho Técnico Instrumentado. A partir destes dados, foi possível estabelecer uma comparação do desempenho entre os alunos que fizeram o curso à distância e os que aprenderam o programa computacional de forma presencial.

\section{APRESENTAÇÃO E DISCUSSÃO DOS RESULTADOS}

Os dados obtidos foram analisados, estatisticamente, através da ferramenta computacional IBM SPSS Statistics, disponibilizado pela universidade. Foi realizado um teste não paramétrico de Kruskall-Wallis, complementado pelo teste de comparações múltiplas. O critério de decisão para os testes foi de nível de significância de $5 \%$. A análise do desempenho dos alunos foi realizada através de histogramas, para uma melhor visualização dos resultados e para efeitos de comparação.

\subsection{Resultados do Grupo 1}

A Figura 2 mostra um histograma, comparando os resultados dos alunos do Grupo 1 com os do Grupo Presencial. A análise da Figura 2 torna evidente que a maioria dos alunos do curso Ead, aproximadamente $74 \%$, obtiveram um desempenho acima de 6 , que é a média necessária para aprovação nas disciplinas da UFRGS. Porém, observase que a comparação entre os dois grupos indica, de uma forma geral, que o grupo presencial teve um desempenho superior. Acredita-se que esta diferença esteja relacionada ao aprendizado da impressão dos desenhos no papel. Os alunos da disciplina presencial puderam praticar a impressão de seus desenhos durante todo 0 semestre letivo. Já os alunos do curso à distância realizaram apenas um exercício de impressão.

Figura 2 - Histograma comparativo Grupo 1 x Grupo Presencial - Avaliação Final

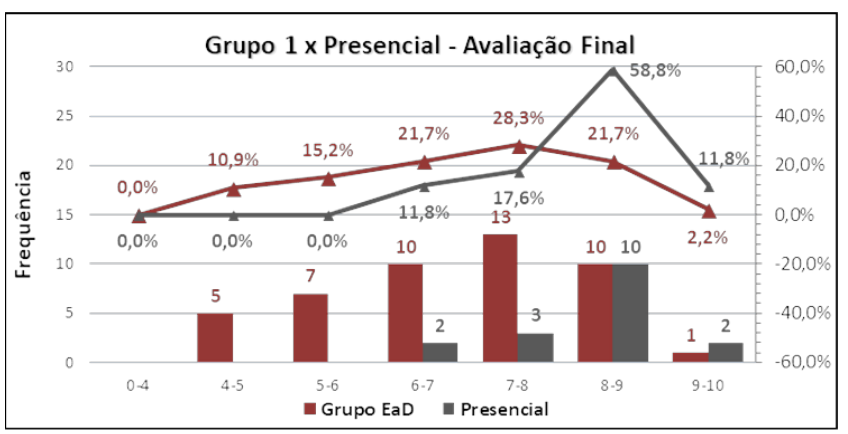

Assim, torna-se importante a avaliação do desempenho dos alunos no trabalho avaliativo, considerando-se, separadamente, os resultados obtidos com o questionário e 
com o exercício prático de impressão. As Figuras 3a e 3b apresentam os histogramas de desempenho do questionário e do exercício prático dos dois grupos estudados separadamente.

Figura 3 - Histogramas Comparativos Grupo EaD x Grupo Presencial (a) Avaliação Questionário e (b) Avaliação Plotagem

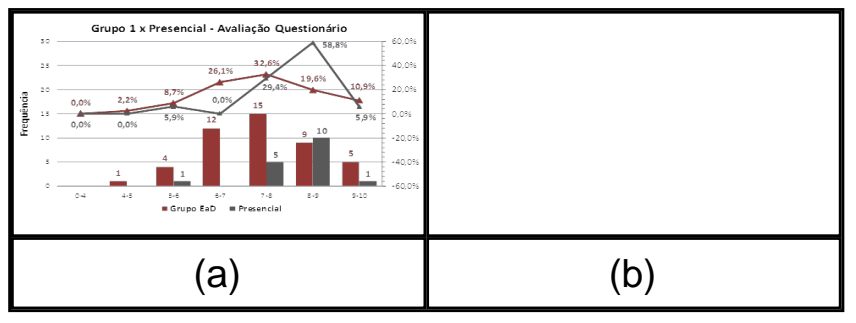

Verifica-se que, de uma forma geral, o desempenho dos dois grupos foi muito semelhante no questionário (Figura 3a), sendo o desempenho dos alunos do curso à distância superior ao dos alunos do curso presencial nas faixas 6-7 e 9-10. Porém, ao analisar-se a Figura 3b, fica evidenciado que o desempenho dos alunos do Grupo 1 no exercício prático sobre impressão foi inferior, pois, observa-se que $50 \%$ dos alunos ficaram abaixo da média 6. Esta constatação torna evidente a necessidade do oferecimento de um maior número de atividades práticas relacionadas ao quesito impressão no curso à distância. A partir desse resultado, optou-se por realizar uma nova implementação do curso EaD, propondo mais exercícios de plotagem.

\subsection{Resultados do Grupo 2}

A partir dos resultados obtidos com o Grupo 1, foram introduzidas novas atividades práticas relacionadas à plotagem no curso. Após a realização do trabalho avaliativo pelo Grupo 2, foi realizada uma nova avaliação estatística para verificação do desempenho desse grupo. A Figura 4 apresenta um histograma comparativo entre os Grupos 1 e 2 referentes ao seu desempenho na avaliação da plotagem.

Figura 4 - Histograma comparativo entre Grupos 1 e 2 - Avaliação de Plotagem 


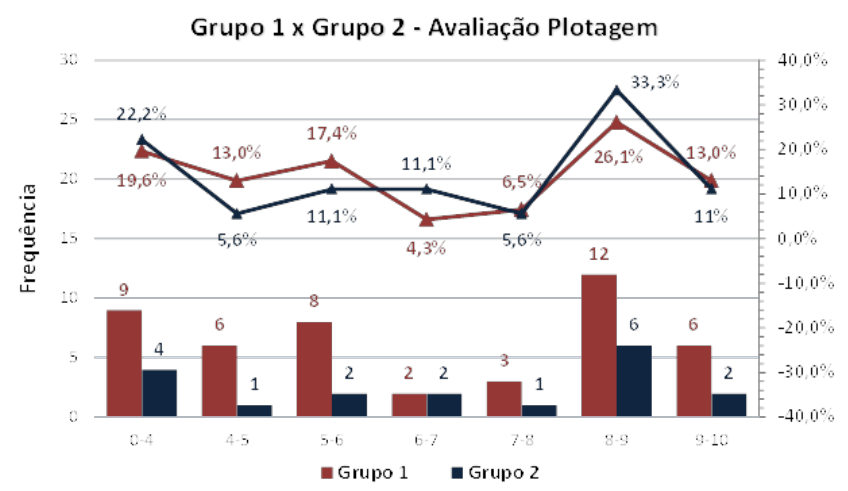

Observa-se que o Grupo 2 apresentou um melhor desempenho em relação ao Grupo 1, onde $61 \%$ dos alunos ficaram acima da média, indicando uma provável eficácia na introdução dos exercícios no curso. Porém, a comparação entre o Grupo 2 e o Grupo Presencial, que pode ser visualizada no histograma da Figura 5, indica que o desempenho do Grupo 2 ainda é inferior ao Grupo Presencial.

Figura 5 - Histograma comparativo Grupo 2 x Grupo Presencial - Avaliação Final

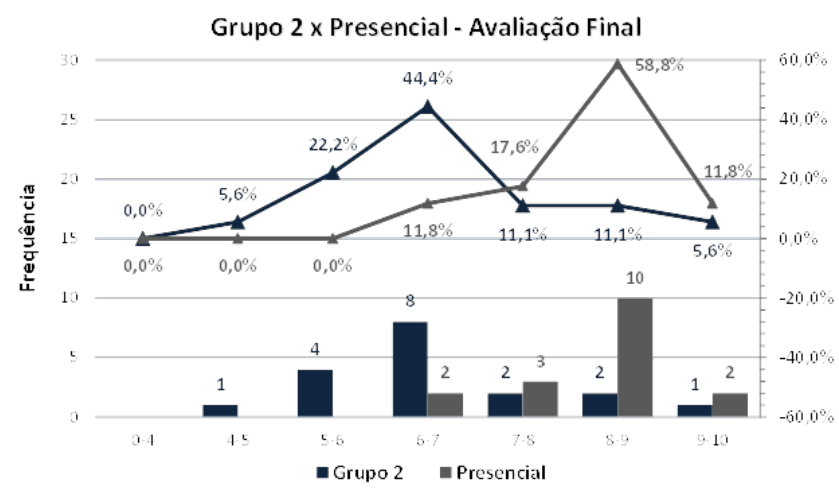

Ao longo da implementação do curso com os dois grupos, foi observado, pela equipe de tutores e professor, através do monitoramento dos alunos, que os estudantes de ambos os grupos demonstraram uma falta de comprometimento e de preparação frente a essa nova proposta de aprendizagem, bem como sua dificuldade de organização para realizálo dentro do prazo estabelecido. A mesma constatação havia sido feita em relação ao Grupo 1. O questionário autoavaliativo respondido pelos dois grupos corroborou estas observações.Este comportamento denota, em geral, uma falta de preparo dos alunos para a realização de um curso à distância, o que, provavelmente, vem a contribuir para um aproveitamento inferior às potencialidades dessa proposta de aprendizagem. A avaliação dos Grupos 1 e 2 indicou a necessidade de auxiliar os alunos na realização de um curso EaD. Acreditou-se que essa assistência teria um reflexo direto no desempenho dos estudantes. Optou-se, então, pela realização de mais uma implementação do curso com um grupo novo de alunos. Esse grupo recebeu a denominação de Grupo 3. 


\subsection{Resultados do Grupo 3}

Antes da implementação do curso com o Grupo 3, a equipe de professor e tutores decidiu elaborar um tutorial, denominado "Manual do Cadinho". Esse manual foi escrito utilizando-se uma linguagem coloquial, procurando, inicialmente, ressaltar o papel do aluno na aprendizagem em um curso à distância. Buscou-se, também, oferecer sugestões que enfatizassem a organização para o estudo, a importância de uma aprendizagem ativa e a relevância da comunicação com os professores e tutores, bem como entre os participantes do curso, através das alternativas disponibilizadas: Fórum e Mensagens dentro do AVA. Finalizado o trabalho avaliativo pelo Grupo 3, foi realizada uma avaliação estatística para verificação do desempenho desse grupo. A Figura 6 mostra um histograma da Avaliação Final do Grupo 3, procurando estabelecer um comparativo com o Grupo Presencial.

Figura 6 - Histograma comparativo Grupo 3 x Grupo Presencial - Avaliação Final

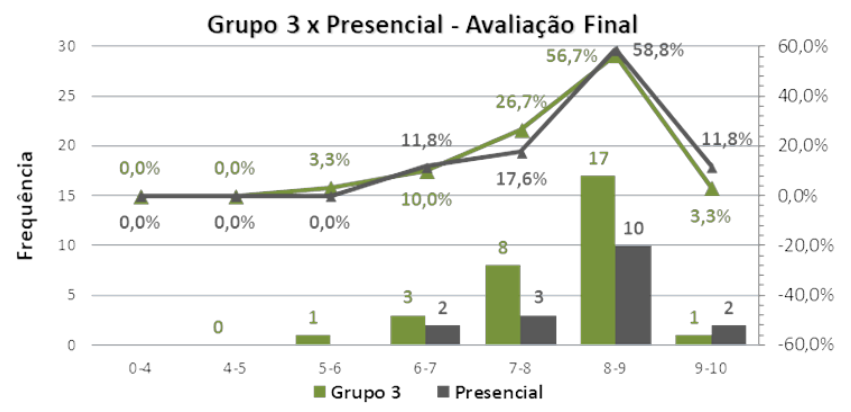

Os resultados indicam um aumento no desempenho total desse grupo em relação aos grupos anteriores, onde é possível perceber que quase a totalidade dos alunos se encontram acima da média (96,7\%). Além disso, observa-se que a maioria dos alunos $(56,7 \%)$ ficou na faixa $8-9$, praticamente o mesmo percentual que os alunos do Grupo Presencial. O grupo de tutores e professor observou uma mudança de atitude dos estudantes em relação ao curso, apresentando uma maior comprometimento e organização para realizá-lo. Acredita-se que as alterações realizadas no curso, bem como a introdução do tutorial tenham contribuído significativamente no aumento do desempenho dos alunos do Grupo 3, bem como para a redução do índice de evasão.

Ao longo da implementação do curso, em todos os grupos, constatou-se uma baixa taxa de comunicação entre seus membros através do Fórum e de Mensagens através do AVA. Segundo MORAN (2014), a colaboração durante o curso é muito importante, pois amplia o conceito de pesquisa significativa, estimulando os alunos a serem pesquisadores e não apenas executores de tarefas, sendo motivados a investigar além do senso comum. $\mathrm{Na}$ continuidade da pesquisa, estuda-se formas de estimular a 
comunicação entre os alunos durante o curso.

\section{CONSIDERAÇÕES FINAIS}

Este trabalho desenvolveu um modelo pedagógico que foi utilizado para a criação de um curso à distância no Ambiente Virtual de Aprendizagem MOODLE UFRGS, cujo objetivo era 0 ensino do programa computacional AutoCAD 2D. As análises estatísticas demonstraram que o desempenho dos alunos que realizaram a última versão do curso à distância foi bastante satisfatório, sendo equiparada ao desempenho dos estudantes que aprenderam a ferramenta de forma presencial. Esses resultados indicam a adequação do curso para o ensino do programa computacional AutoCAD 2D a distância. Porém, observou-se uma baixa taxa de comunicação entre os alunos durante a realização do curso. Na continuidade do trabalho, procurar-se-á alternativas para solucionar esta questão. Acredita-se que uma maior comunicação entre o grupo de aprendizagem, gere debates que contribuam para um crescimento cognitivo do aluno, resultando em seu melhor desempenho.

\section{REFERÊNCIAS}

BEHAR, P.A et al. Modelos pedagógicos em Educação a Distância. Porto Alegre: Artmed, 2009.

BERNARDI, M. Prática pedagógica em ead: uma proposta de arquitetura pedagógica para formação continuada de professores. 2011. 200f. Tese (Doutorado em Engenharia) - Faculdade de Educação, Universidade Federal do Rio Grande do Sul, Porto Alegre, 2011.

LAPA, A. B. Introdução à Educação à Distância. Disponível em: http://www.libras.ufsc.br/ hiperlab/avalibras/moodle/prelogin/adl/fb/logs/Arquivos/textos/intro_ead/Intro_EAD_pdf_. pdf. Acesso em: maio 2017

MOORE, M.G.; KEARSLEY, G. Distance education: a systems view of online learning. Wadsworth: Cengage Learning, 2011.

MORAN, J.M. O A educação à distância, mais focada em pesquisa e colaboração.2014. Disponívelem:<http://www.eca.usp.br/prof/moran/site/textos/educacao_online/pesquisa e_colaboracao.pdf>Acesso em: maio 2014 